\title{
Voriconazole: A Review of Population Pharmacokinetic Analyses
}

\author{
Changcheng Shi ${ }^{1} \cdot$ Yubo Xiao $^{2} \cdot$ Yong Mao $^{1} \cdot$ Jing Wu ${ }^{3} \cdot$ Nengming Lin $^{4}$
}

Published online: 28 January 2019

(c) The Author(s) 2019

\begin{abstract}
Numerous population pharmacokinetic studies on voriconazole have been conducted in recent years. This review aimed to comprehensively summarize the population pharmacokinetic models for voriconazole and to determine which covariates have been identified and which remain to be explored. We searched the PubMed and EMBASE databases from inception to March 2018 for population pharmacokinetic analyses of voriconazole using the nonlinear mixed-effect method. A total of 16 studies were included in this review, of which 11 models were described in adult populations, four were described in pediatric populations, and the remaining study included both adult and pediatric populations. The absorption profiles of voriconazole in both adult and pediatric studies were best described as first-order absorption models. The typical distribution volumes were similar in adult and pediatric patients, but the estimated clearances in pediatric patients were significantly higher than those in adult patients. The most commonly identified covariates were body weight, the cytochrome P450 2C19 genotype, liver function, and concomitant medications. Only one study evaluated the model using an external method. Further population pharmacokinetic studies on pediatric patients aged younger than 2 years are warranted. Furthermore, new or controversial potential covariates, such as inflammation, the cytochrome P450 3A4 genotype, concomitant medications (particularly various types and dosages of proton pump inhibitors and glucocorticoids), and various measures of body weight, should be tested because the unexplained variability remains relatively high. In addition, previously published models should be externally evaluated, and the predictive performance of the various models should be compared.
\end{abstract}

Changcheng Shi and Yubo Xiao contributed equally to this work and should be considered co-first authors.

Nengming Lin

lnm1013@163.com

1 Department of Clinical Pharmacy, Affiliated Hangzhou First People's Hospital, Zhejiang University School of Medicine, Hangzhou, China

2 Department of Pharmacometrics, Mosim Co., Ltd, Shanghai, China

3 Department of Pharmacy, Zhejiang Pharmaceutical College, Ningbo, China

4 Department of Clinical Pharmacology, Translational Medicine Research Center, Affiliated Hangzhou First People's Hospital, Zhejiang University School of Medicine, No. 261 Huansha Road, Hangzhou 310006, China

\section{Key Points}

The final structural population pharmacokinetic models of voriconazole differ between adult and pediatric populations.

Potential and controversial covariates, such as inflammation, the cytochrome P450 3A4 genotype, concomitant medications, and various measures of body weight, should be tested in future studies because the unexplained variability remains relatively high.

Previously published models should be externally evaluated, and the predictive performances of the models should be compared.

\section{Introduction}

Voriconazole is a new-generation triazole antifungal agent with potent activity against a wide range of clinically significant pathogens, including Aspergillus and Candida, as 
well as some less common fungal pathogens [1]. Since its approval in 2002, voriconazole has changed the approach to the management of invasive fungal diseases. The Infectious Diseases Society of America guidelines now recommend voriconazole as the first-line drug for the treatment of invasive aspergillosis and as an alternative drug for the treatment of candidemia [2, 3].

In recent years, numerous studies have investigated the exposure-response relationship of voriconazole. The findings from these studies established that low concentrations might result in higher rates of treatment failure, whereas higher concentrations are associated with increased toxicity; thus, the results identify a narrow target trough concentration range for voriconazole $[4,5]$. Furthermore, the wide inter- and intraindividual pharmacokinetic variability is of great concern.

Several factors are reportedly associated with the large variability in the exposure to conventional doses of voriconazole, and these include the nonlinear pharmacokinetic properties of voriconazole, the cytochrome P450 (CYP) 2C19 genotype, hepatic dysfunction, and drug interactions [6]. Therapeutic drug monitoring (TDM) for voriconazole is recommended for the optimizing outcomes and reducing toxicity in clinical practice [7]. However, the TDM method can be implemented only after treatment has been initiated, and the samples for TDM are traditionally procured at steady state. In fact, steady-state trough concentrations are reached approximately 5 days after standard administration. Although the steady state can be reached $24 \mathrm{~h}$ after the administration of a loading dose, a waiting time is still needed and might contribute to a worse prognosis [6]. Therefore, the identification of factors that contribute to the high variability in voriconazole pharmacokinetics is important for determining the appropriate dosage as early as possible.

Population pharmacokinetic modeling is widely used in the field of clinical pharmacology because it helps determine the typical pharmacokinetic parameters of a population and can be used to obtain the sources of pharmacokinetic variability [8]. The integration of the population pharmacokinetic model with the Bayesian forecasting method can help guide dosage adjustments based on a limited number of drug concentration measurements [9]. Indeed, many population pharmacokinetic studies on voriconazole have been conducted over the last decade. This review provides an overview of the published studies on the population pharmacokinetics of voriconazole. The objective was to provide a systematic comparison of the population pharmacokinetic models published for voriconazole and to determine which covariates have been identified and which remain to be explored.

\section{Methods}

\subsection{Search Strategy}

The PubMed and EMBASE databases were searched from inception to March 2018 using the following search terms: 'voriconazole' AND ('population pharmacokinetic' OR 'pharmacometrics' OR 'pharmacokinetic model' OR 'popPK' OR 'pop PK' OR 'PPK' OR 'nonlinear mixed effect model' OR 'NONMEM'). The reference lists of the relevant studies were searched for additional literature.

\subsection{Inclusion/Exclusion Criteria}

We included all described population pharmacokinetic models for voriconazole. The studies needed to meet the following criteria for inclusion in this review: (1) studied populations, pediatric and adult patients or healthy volunteers; (2) treatment, voriconazole was used as the study drug, regardless of whether it was administered intravenously or orally; and (3) pharmacokinetic analysis, a nonlinear, mixedeffect population pharmacokinetic modeling approach was employed. The following studies were excluded: (1) reviews, methodology articles, and in vitro and animal studies; (2) papers not written in English; and (3) studies that used noncompartmental or nonparametric approaches.

\subsection{Data Extraction}

Two authors independently performed data extraction using a data collection form, and any discrepancies were resolved by discussion. The following variables were recorded from the identified studies: first author, year of publication, number of patients, patient characteristics (age, sex, weight, genotype, and pathology), route of administration, observed voriconazole concentration, method used for voriconazole determination, number of observations, observations per patient, data source, software used for modeling, dosing simulations, structural and statistical model, tested and retained covariates, and model evaluation method. The model evaluation methods were divided into three types based on the increasing order of quality: basic internal, advanced internal, and external model evaluation [10].

\section{Results}

The initial database search yielded 152 publications, and after selection, a total of 16 studies involving 1411 participants met the inclusion criteria [11-26]. The population characteristics of the included studies are summarized in 


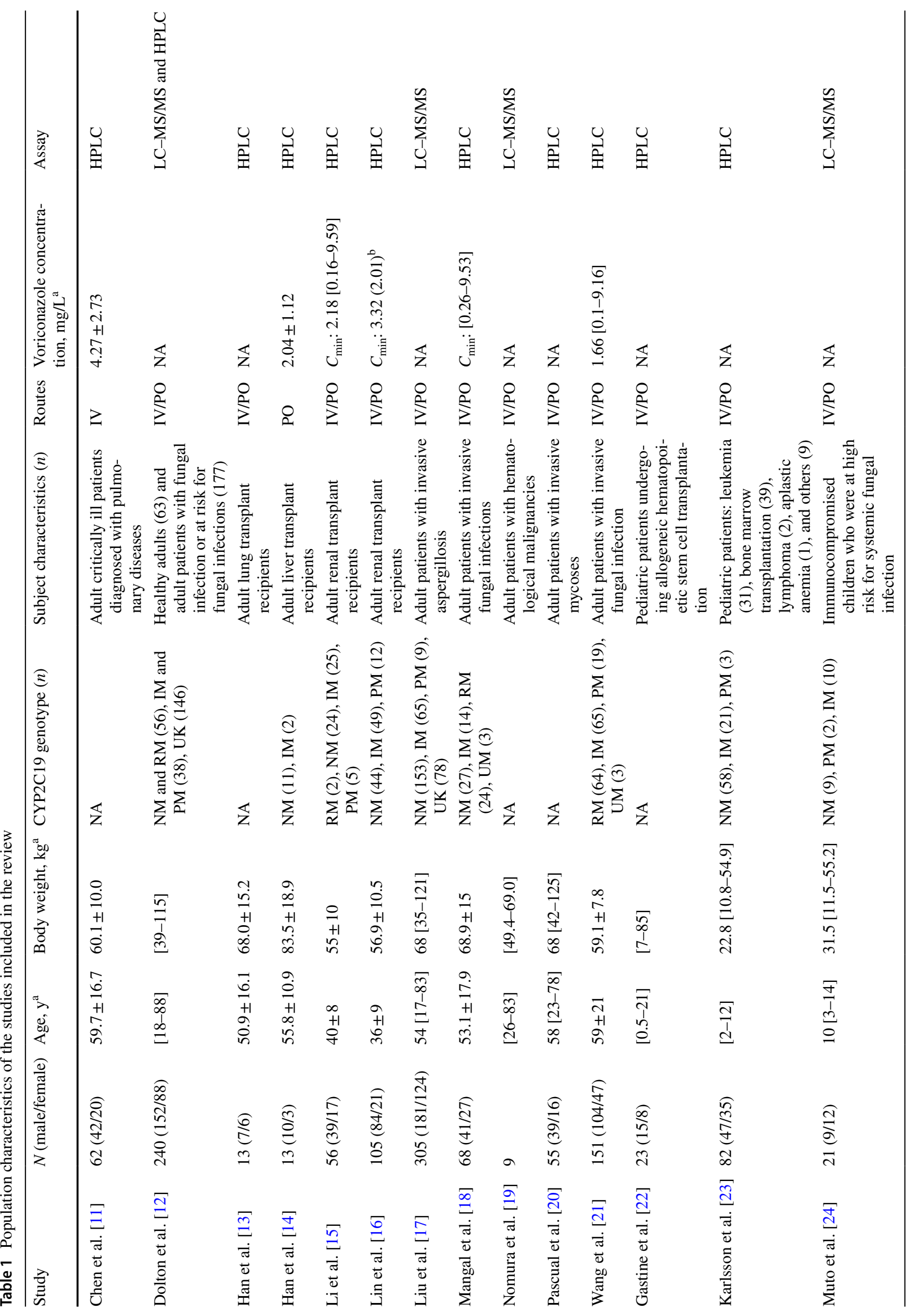




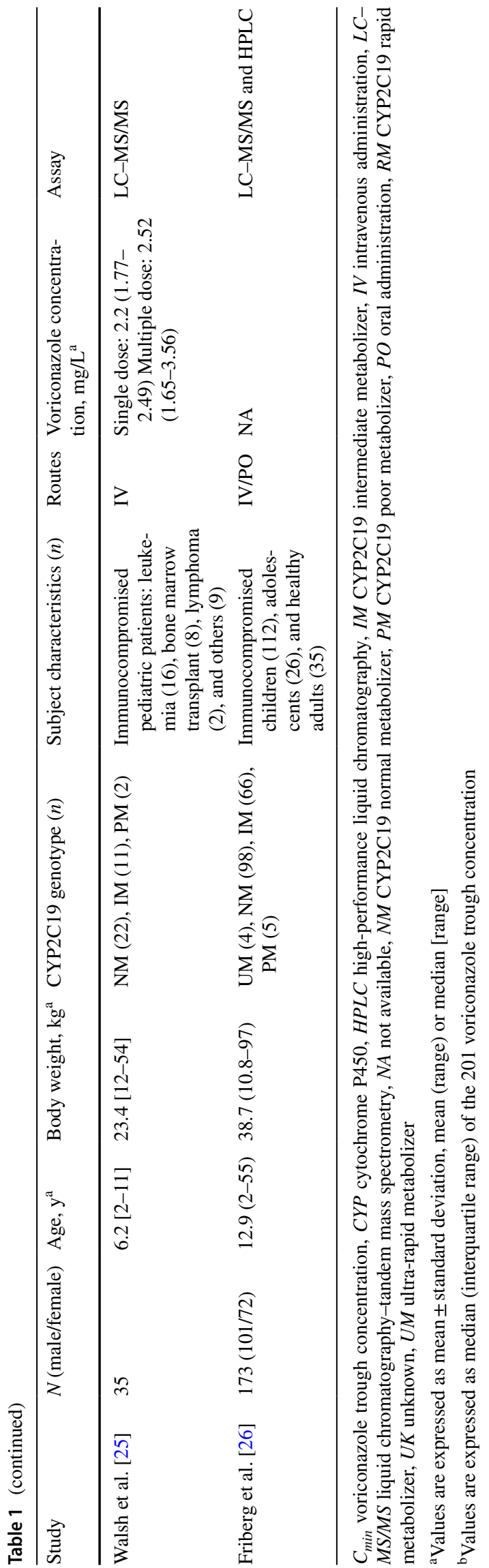

Table 1. The years of publication ranged from 2004 to 2018 . The number of participants included in each study ranged from 9 to 305 (median: 59), and ten studies (62.5\%) included more than 50 participants. CYP 2C19 genotyping data were included in 11 articles [12, 14-18, 21, 23-26]. Among the 16 publications describing a population pharmacokinetic model for voriconazole, 11 described studies conducted in adult participants, [11-21] whereas four of the studies were conducted in pediatric populations, [22-25] and the remaining study by Friberg et al. [26] included both adult and pediatric patients. The studied populations consisted of healthy volunteers and patients who were administered voriconazole for the treatment or prophylaxis of fungal infections, possibly accompanied by additional pathologies, including pulmonary diseases, organ transplant, and hematological malignancies. Both intravenous and oral formulations were administered in all but three of the included studies, and in the remaining three studies, only intravenous [11, 25] or oral [14] formulations were used. Seven publications reported the means or medians of the observed voriconazole concentrations [11, 14-16, 18, 21, 25]. In all the included studies, a high-performance liquid chromatography was employed for the determination of the voriconazole concentration.

The model characteristics of the included studies are summarized in Table 2 . The number of observations ranged from 36 to 3352 (median 342), and the median observations per patient was nine. In addition, $56 \%$ of the studies involved rich data, and only two studies $[15,18]$ involved sparse data from routine TDM practice. Almost all the included studies utilized the gold-standard software NONMEM to construct a population pharmacokinetic model with the exception of two studies, which used Phoenix NLME software [15, 16]. All the models were validated using various advanced internal methods, including bootstrap, [11,13-16, 18-21, 26] visual predictive check or corrected visual predictive check, [11, $12,14,17,22,24,26]$ case deletion diagnostics, [23] and cross-validation [25]. Only one study performed an external evaluation using a separate cohort [14]. Simulation analyses were also performed in ten studies to determine the optimal dosing regimens $[11,13,16,18-23,26]$. The majority of the studies adopted the trough concentration as the target, and the remaining studies chose the free area under the plasma concentration-time curve from 0 to $24 \mathrm{~h}$ divided by the minimum inhibitory concentration, trough concentration/ minimum inhibitory concentration, and the reference adult area under the plasma concentration-time curve distribution.

The final structural model, pharmacokinetic parameters, model variability, covariates tested, and covariates retained in the final model are summarized in Table 3. The absorption characteristics of voriconazole were described as a firstorder process in 13 of the included studies [12-18, 20-24, 26]. The absorption rate constant was fixed to the literature value in six studies [15-18,21, 22], and a lag time was used 


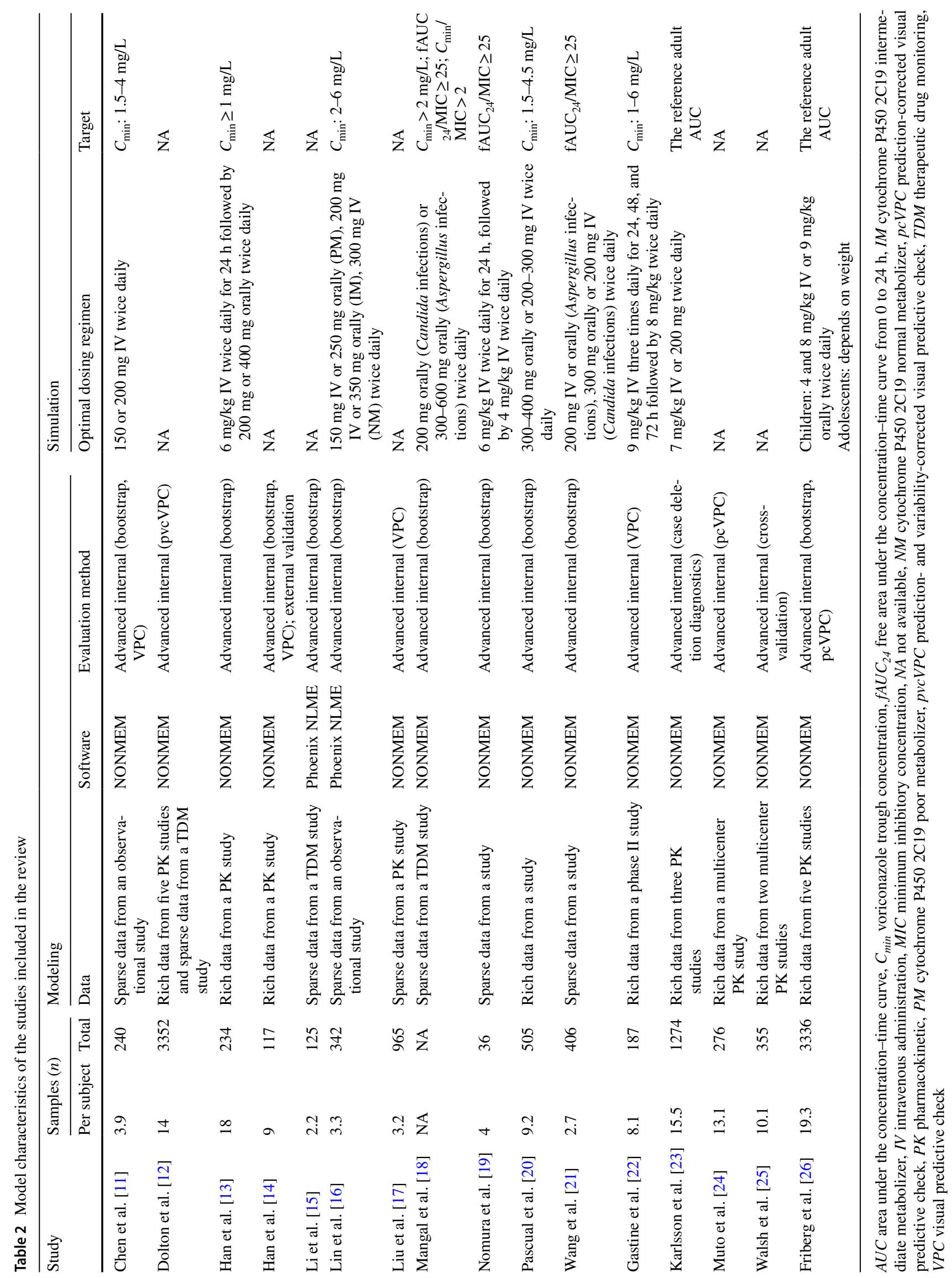


in five of the included studies $[12,14,17,24,26]$ to characterize delayed absorption. The typical oral bioavailability of voriconazole reportedly ranged from $45.9 \%$ to $94.2 \%$ in adult patients $(n=6)$ and from $44.6 \%$ to $64.5 \%$ in pediatric populations $(n=4)$.

In adults, the population pharmacokinetics of voriconazole were best described by a one-compartment model in eight studies [11, 14-16, 18-21] and by a two-compartment model in three studies $[12,13,17]$. The median (range) estimated value of the distribution volume $(V)$ was $77.6 \mathrm{~L}$ (27.1-200 L) [ $n=9]$. Most of the studies conducted in adult populations described the elimination of voriconazole as linear elimination, [11, 13-16, 19-21] and the median (range) estimated value for the linear clearance $\left(\mathrm{CL}_{\mathrm{L}}\right)$ was $5.25 \mathrm{~L} / \mathrm{h}$ $(3.45-11.2 \mathrm{~L} / \mathrm{h})[n=8]$. All of the studies conducted in pediatric populations employed a two-compartment model with various types of elimination, including linear, [25] nonlinear, $[22,23]$ and mixed linear and nonlinear elimination $[24,26]$. The median (range) estimated values for the central distribution volume $\left(V_{1}\right)$ were $1.07 \mathrm{~L} / \mathrm{kg}(0.81-3.26 \mathrm{~L} / \mathrm{kg})[n=5]$. The median (range) estimated values for the maximum voriconazole metabolic rate $\left(V_{\max }\right)$ and the Michaelis-Menten constant were $0.957 \mathrm{mg} / \mathrm{h} / \mathrm{kg}(0.341-1.178 \mathrm{mg} / \mathrm{h} / \mathrm{kg})[n=4]$ and $1.15 \mathrm{mg} / \mathrm{L}(0.922-3.03 \mathrm{mg} / \mathrm{L})[n=4]$, respectively. The total clearance (CL) values for increasing voriconazole concentration predicted with the different models were compared, and the results are shown in Fig. 1.

Between-subject variability (BSV) is commonly described by an exponential model. The BSV in bioavailability was estimated using additive random effects on a logit scale in five studies [17, 20, 22, 24, 26]. In adult patients, the median (range) BSV in $V$ (or $V_{1}$ ) and $\mathrm{CL}_{\mathrm{L}}$ were $32.75 \%$ (12-98\%) [ $n=8$ ] and 41\% (21.3-107\%) [ $n=8]$, respectively, and the median (range) BSV in $V$ (or $V_{1}$ ) and $\mathrm{CL}_{\mathrm{L}}$ in pediatric populations was $14.2 \%(13.6-45.4 \%)[n=3]$ and 69.6\% (66.5-117.4\%) [ $n=3]$, respectively. Only one study estimated the between-occasion variability in intrinsic CL and obtained a value of $43 \%$ [24].

A proportional residual error model was most commonly used to describe residual variability, $[11,15,17,18,20,23$, $24,26]$ and the residual variability obtained using a proportional model ranged from $13 \%$ to $61 \%$. Notably, half of the residual variability values were modeled as additive errors on the log-transformed concentrations, which approximately corresponded to a proportional error on untransformed data $[17,23,24,26]$. Five studies used a combined model residual error model and the median (range) values were $0.016 \mathrm{mg} / \mathrm{L}$ $(0.005-0.49 \mathrm{mg} / \mathrm{L})$ and $33.8 \%(10.8-43 \%)$ [12-14, 21, 22]. Only the study conducted by Lin et al. [16] used an additive residual error model, and the value was $0.57 \mathrm{mg} / \mathrm{L}$.

Numerous factors were tested in the modeling process, and the most commonly identified covariates were body weight, the CYP2C19 genotype, liver function, and concomitant medications. For adult populations, the covariates identified in the population pharmacokinetic studies of voriconazole included body weight, the CYP2C19 genotype, postoperative time, direct bilirubin, the international normalized ratio, aspartate transaminase, alkaline phosphatase, severe cholestasis, concomitant medications, cystic fibrosis, and age. In contrast, the studies on pediatric populations identified the following covariates: body weight, the CYP2C19 genotype, alanine transaminase, alkaline phosphatase, and the study population (adolescent or child).

\section{Discussion}

Population pharmacokinetic modeling methods can be statistically classified as either parametric or nonparametric. The main difference between parametric and nonparametric methods is that the former assumes that the parameter and error distributions follow normal, or log-normal, distributions, whereas, nonparametric methods make no assumption regarding the shapes of the underlying parameter distributions [27]. To the best of our knowledge, only two population pharmacokinetic models of voriconazole obtained using a nonparametric approach have been published to date [28, 29]. We focus on the parametric approach in this review. It remains unclear which approach is more suitable for voriconazole therapy in a specific population, and more studies comparing both methods are warranted.

In 2016, McDougall et al. [30] published a hybrid model for voriconazole that integrated information from prior population pharmacokinetic models. The authors identified and briefly reviewed nine population pharmacokinetic studies on voriconazole. After that publication, an increasing number of publications have focused on this topic. In the current review, we summarized a total of 16 parametric population studies on voriconazole.

The majority of publications in this field have included adult organ transplant recipients and immunocompromised pediatric patients. Notably, no published population analysis of voriconazole has included pediatric patients aged younger than 2 years, potentially because voriconazole has officially only been approved for adults and pediatric patients aged $\geq 2$ years. Nevertheless, voriconazole has commonly been administered to this specific population in clinical practice, as summarized in the review by Kadam and Van Den Anker [31]. A recent large-sample retrospective study showed that voriconazole exposure is highly variable in pediatric patients aged younger than 2 years, and the therapeutic range was not achieved in a substantial proportion of the pediatric patients [32]. Therefore, further population pharmacokinetic analyses focusing on this specific population are required. 

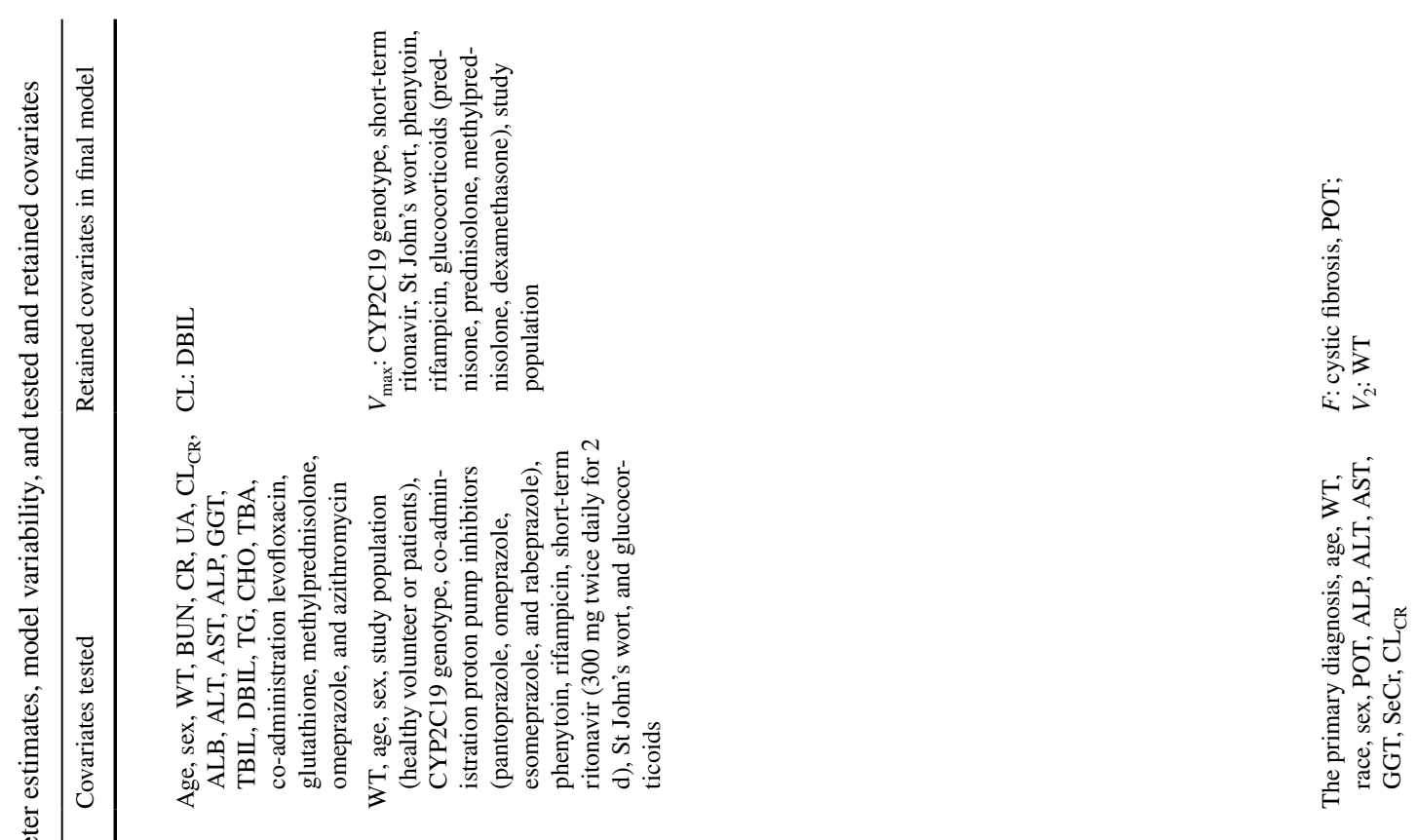$$
\text { (2) }
$$

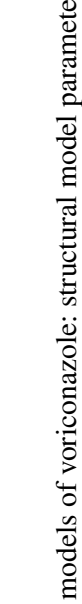




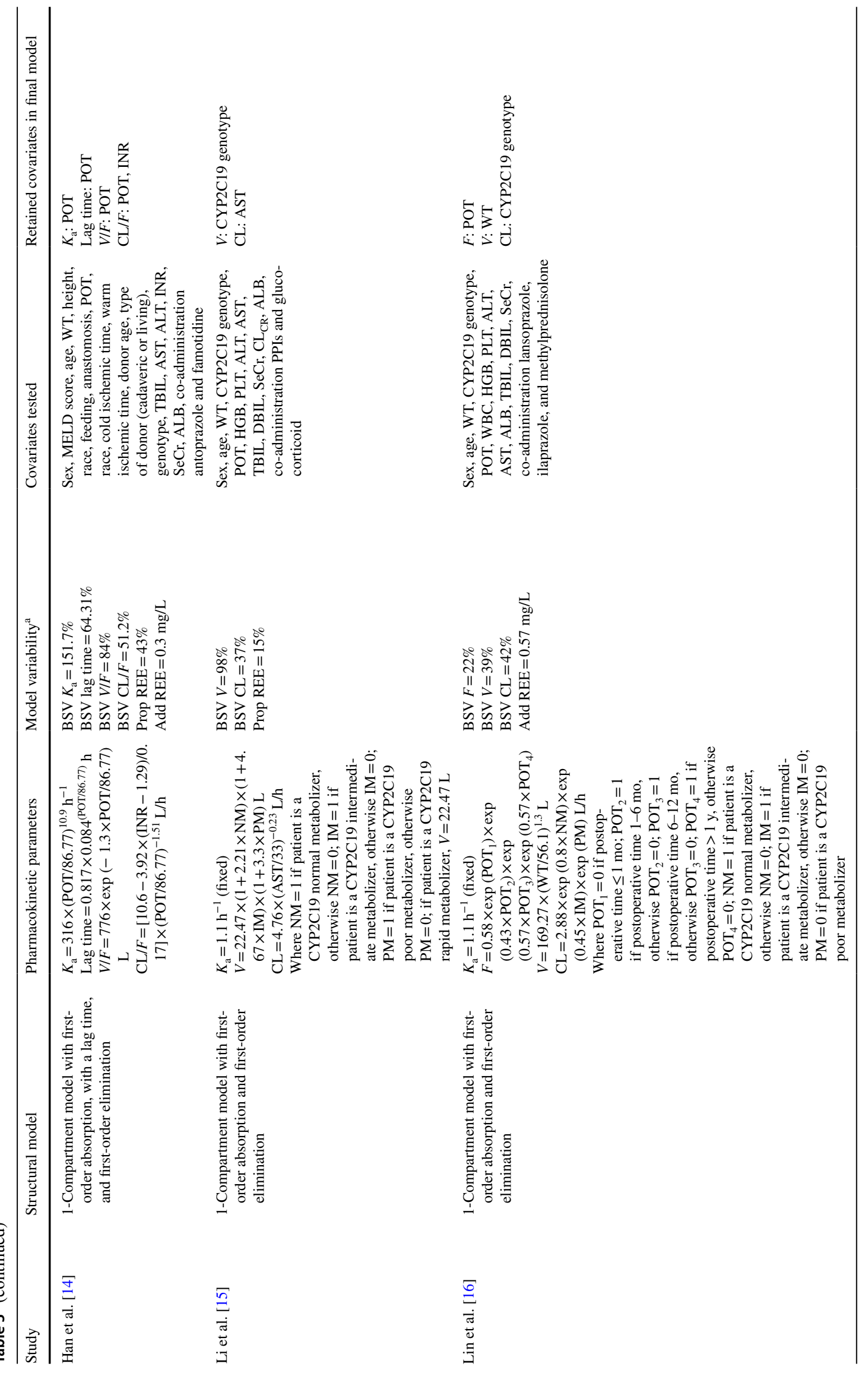




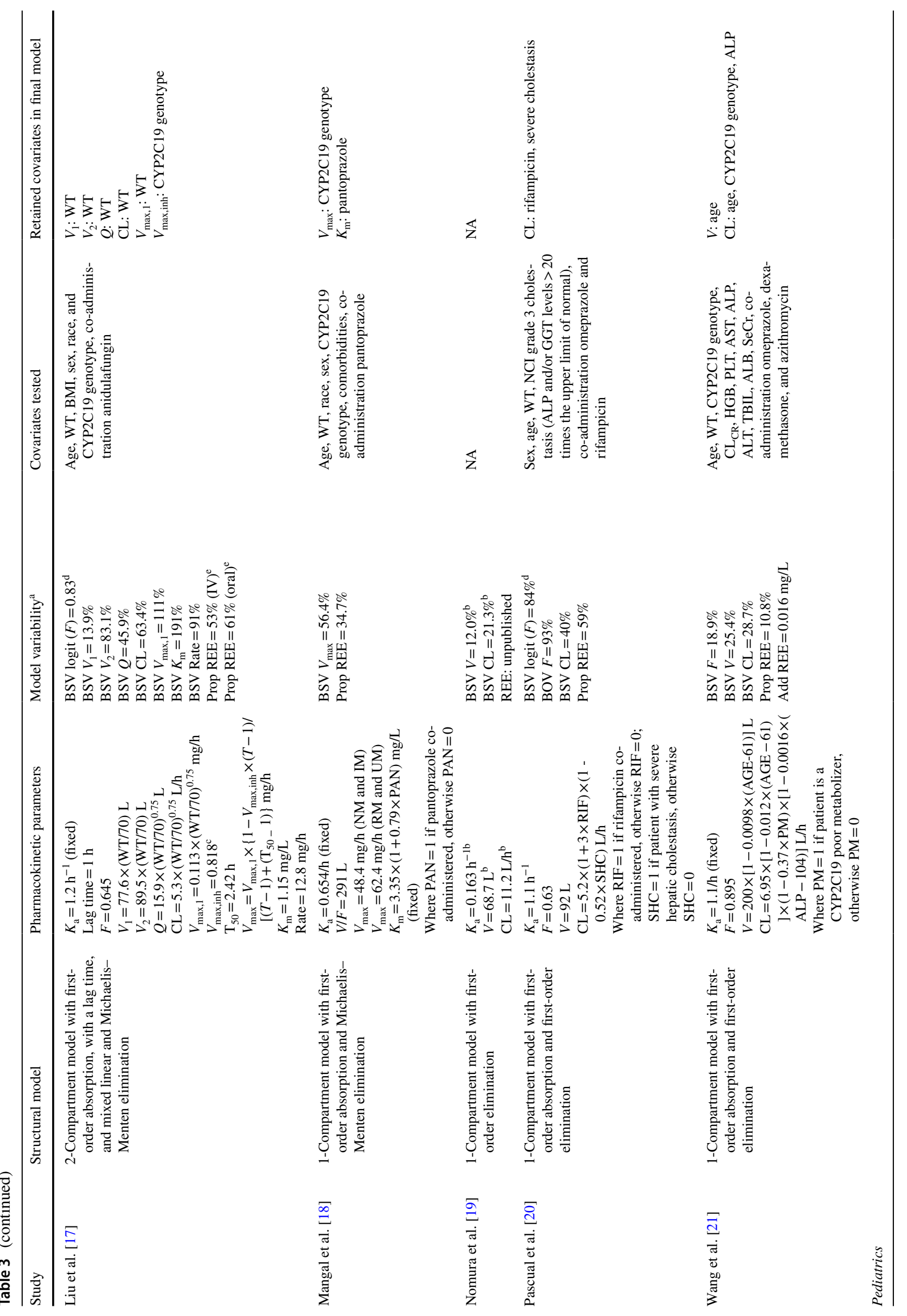




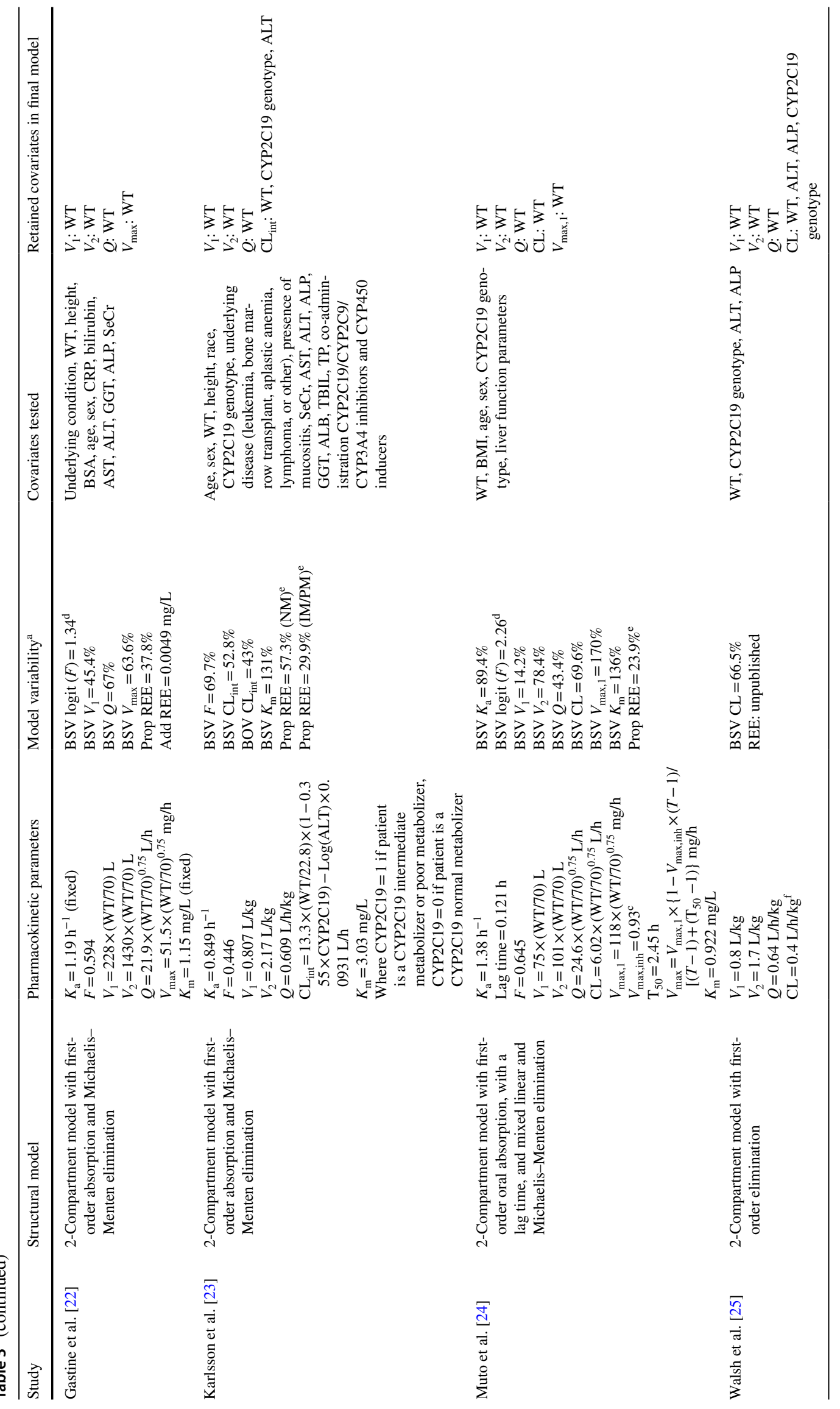




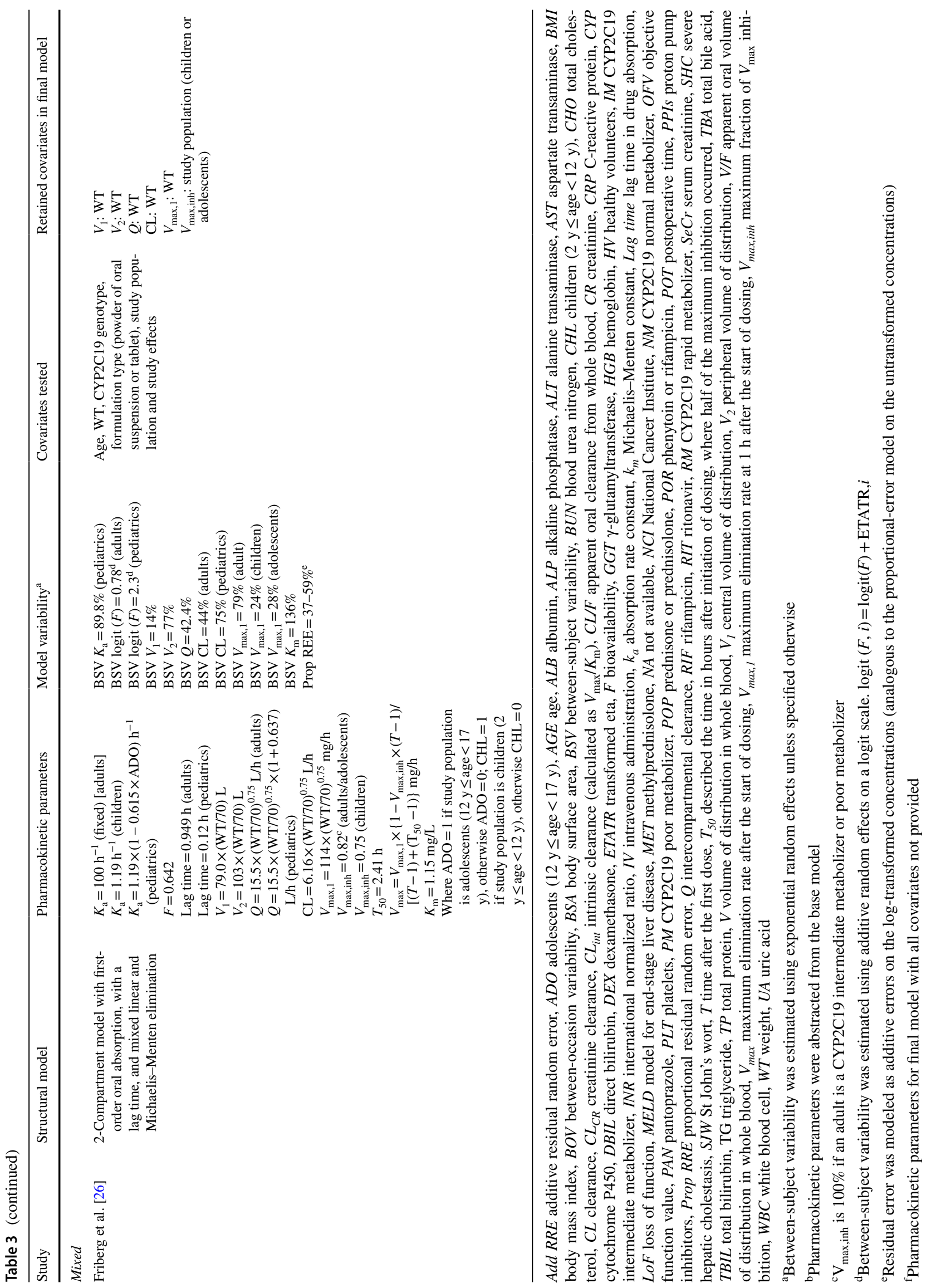


Voriconazole is available in both intravenous and oral forms. The absorption profiles of voriconazole in both adult and pediatric populations have been best described by firstorder absorption models. Nevertheless, the final structural pharmacokinetic models of voriconazole differ between pediatric and adult populations. All the studies conducted in pediatric populations employed a two-compartment model with various types of elimination (linear, nonlinear, or mixed linear and nonlinear elimination). However, the structural model used in most of the studies conducted in adults was a one-compartment model with linear elimination. Notably, two studies on adult patients [15, 18] established a onecompartment model using data from routine TDM practice, which might have resulted in the inability to identify twocompartmental models. Regardless of the patient populations, voriconazole CL was described as a linear process in most of the studies $(n=11)$, which was inconsistent with the nonlinear pharmacokinetic characteristics related to saturable CL mechanisms. In fact, this finding was supported by the results of a comparative study conducted by Farkas et al., [33] who evaluated the accuracy and precision of the predictions of three different structural models (linear, nonlinear, or mixed linear and nonlinear) for voriconazole and found that the linear model was the most accurate. The favorable performance of the linear model might be explained by the applied doses of voriconazole. Although the doses of voriconazole varied among the different studies and populations, the mean or median values of the observed voriconazole concentrations reported in the included studies were not high, ranging from 1.66 to $4.27 \mathrm{mg} / \mathrm{L}$. The nonlinear component of the elimination model might not be pronounced during low-to-moderate voriconazole exposure.

Based on data from 207 healthy participants, the oral bioavailability of voriconazole is more than $90 \%$ [6]. However, the typical bioavailabilities estimated in most of the included population pharmacokinetic studies, particularly in adult organ transplant recipients after transplant surgery and pediatric patients, were relatively lower than those observed in healthy participants. Lin et al. [16] showed that the typical bioavailability value equaled $58 \%$ within 1 month after renal transplantation. Similarly, Han et al. [13] reported that the population estimate of bioavailability in lung transplant populations was only $45.9 \%$. However, both research groups revealed that bioavailability was significantly increased with increases in the postoperative time. Thus, the low bioavailability obtained in the studies could be partially explained by gastrointestinal complications soon after the operation, which are frequently observed in transplant populations $[34,35]$. In addition, specific pathologies, such as cystic fibrosis and mucositis, are associated with poor bioavailability, which should be considered in clinical practice [13, 20]. In the pediatric populations, the median (range) bioavailability equals $61.8 \%$ (range $44.6-64.5 \%$ ) [ $n=4]$, and pediatric patients exhibit significantly decreased bioavailability compared with adults (with the exception of transplant populations). Although several potential covariates were tested, none were found to have a significant effect on bioavailability in pediatric patients. A physiologically based pharmacokinetic study suggested that the lower bioavailability of voriconazole observed in pediatric patients compared with adults might be related to intestinal first-pass metabolism [36]. In addition, the diet might contribute to the different bioavailabilities between pediatric and adult patients. It is well known that diet reduced the effects of exposure to voriconazole, [6] and adults can generally better control their diet.

The estimated values for $V$ (or $V_{1}$ ) were similar among the included studies. However, as demonstrated in Fig. 1, the predicted total CL in pediatric patients was significantly higher than that in adult patients. Moreover, the BSV in CL was greater in pediatric patients than in adult patients. Voriconazole is metabolized by drug-metabolizing enzymes, and gene expression and enzyme activity are known to change with age. An in vitro study showed that oxidative enzymes derived from pediatric patients aged 2-8 years metabolized voriconazole at a three-fold higher rate than those derived from adults [37]. The researchers revealed that CYP2C19 and flavin-containing monooxygenase 3 play notably more important roles than CYP3A4 in the elimination of voriconazole in children [37]. A recent study conducted by Zane et al. [38] quantified the protein expression of CYP2C19 in pediatric and adult hepatic tissues and revealed that the protein expression of CYP2C19 was approximately two-fold higher in pediatric than in adult hepatic tissue. Moreover, investigators revealed that CYP2C19 activity at birth was only $26 \%$ of that observed in adults. The CYP2C19 activity rapidly increases up to approximately two-fold higher than the value in adults during the first year after birth, and the CYP2C19 activity from 1 to 5 years of age is approximately $160 \%$ of that observed in adults and then decreases slowly until it reaches the level observed in adults at 10 years of age [39]. Thus, the ontogeny of protein expression and enzyme activity might contribute to the differences in CL values obtained between pediatric and adult populations.

The dose regimens for voriconazole are based on the body weight at the time of the prescription, which indicates that body weight might be a major source of pharmacokinetic variability. All identified models for pediatric populations incorporated body weight in the CL and distribution parameters. However, only the study conducted by Liu and Mould [17] showed a significant relationship between body weight and CL in adult patients, but the authors also emphasized that the magnitude of the changes in voriconazole exposure associated with body weight was very slight in adults. Moreover, Han et al. [13] performed a simulation analysis on adults and investigated the performances of two dosing 


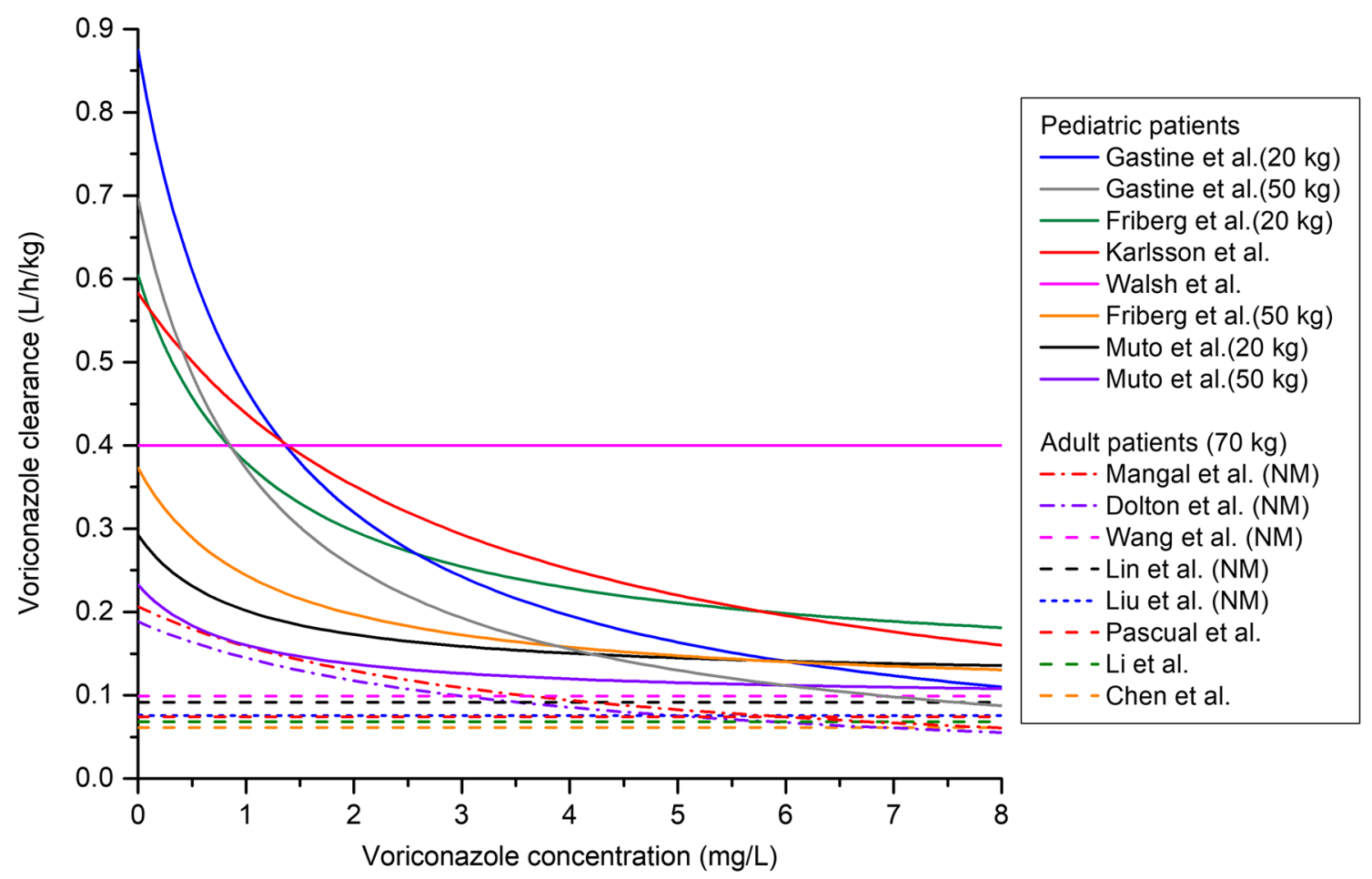

Fig. 1 Comparisons of the predicted voriconazole clearance values in the included studies for increasing concentrations

regimens (fixed and body weight-based dosing) on reducing pharmacokinetic variability, and the results reveled that body weight-based dosing did not decrease the pharmacokinetic variability compared with a fixed-dose strategy. Overall, the lack of effect of body weight on voriconazole elimination does not support the use of a body weight-based dosing strategy for the administration of voriconazole to adult patients. In fact, this finding was supported by the results of several studies that focused on obese patients. These studies revealed high serum concentrations in overweight patients based on the actual body weight [40] and comparable exposure between overweight and normal subjects administered a fixed dose independent of the subject's weight [41]. Nevertheless, it should be mentioned that all the included studies tested only the total body weight and not other measures of body weight, such as ideal body weight (IBW) and adjusted body weight $(\mathrm{ABW})$. A previous study compared voriconazole concentrations in obese patients given a dose of $4 \mathrm{mg} /$ $\mathrm{kg}$ according to their actual body weight, IBW, and ABW [42]. The results indicated that a dosing strategy for voriconazole based on the IBW or ABW might be appropriate [42]. Therefore, the various measures of body weight should be tested in future population analyses.

Voriconazole is mainly metabolized by the CYP2C19 enzyme [6]. Therefore, polymorphisms of the CYP2C19 gene encoding CYP2C19 isoenzymes might be a major source of the variability in the pharmacokinetics of voriconazole. According to the Clinical Pharmacogenetics Implementation Consortium guidelines, five types of CYP2C19 metabolizer phenotypes have been classified: normal metabolizer, intermediate metabolizer (IM), poor metabolizer (PM), rapid metabolizer (RM), and ultra-rapid metabolizer [43]. It should be mentioned that several studies included in this review used the terms "extensive metabolizer" and "heterozygous extensive metabolizer", and these have been replaced by the terms "normal metabolizer" and "intermediate metabolizer", respectively, based on the Clinical Pharmacogenetics Implementation Consortium guidelines. Most of the studies included in the current review retained the CYP2C19 genotype as a significant covariate in the final model. Therefore, genetic testing should be encouraged if appropriate in clinical practice.

For pediatric patients, Karlsson et al. [23] reported that the intrinsic CL of voriconazole is significantly lower in CYP2C19 IM and PM compared with CYP2C19 normal metabolizer. Similarly, for adults, Wang et al. [21] reported that the CL in patients with CYP2C19 PM was 37\% lower compared with those in other genotypes. Dolton et al. [12] found that participants with CYP2C19 IM and PM had a $V_{\max }$ that was $41.2 \%$ lower than that of participants with no loss-of-function alleles. Mangal et al. [18] found that the $V_{\max }$ in adult patients with CYP2C19 RM and ultra-rapid metabolizer was $9 \%$ higher compared with that in patients with CYP2C19 normal metabolizer and IM. Moreover, the 
CYP2C19 genotype can significantly affect both CL [16] and $V[15]$ in renal translation recipients. Nevertheless, few studies have tested other drug-metabolizing enzymes as factors in model building. Indeed, voriconazole is eliminated by not only CYP2C19 but also other drug-metabolizing enzymes, specifically CYP3A4 [6]. To date, several studies have found that genetic variants of CYP3A4 can influence voriconazole exposure [44-46]. Thus, the influence of CYP3A4 polymorphisms should be considered in future population pharmacokinetic studies.

Numerous studies included in the current review demonstrated that reduced voriconazole elimination is significantly associated with impaired liver function, as indicated by elevated alanine transaminase, [24] aspartate transaminase, [15] direct bilirubin, [11] alkaline phosphatase, [21] and international normalized ratio [14] levels. Moreover, Pascual et al. found significantly reduced elimination in adult patients with severe cholestasis [20]. The impact of trough concentrations of voriconazole on hepatotoxicity has been identified. A meta-analysis showed that the incidence of hepatotoxicity increases from $4.2 \%$ for lower serum concentrations to $12.4 \%$ for supratherapeutic concentrations [47]. High trough concentrations of voriconazole can lead to liver injury, and the consequent liver dysfunction will result in metabolic disorders and higher voriconazole exposure. This phenomenon might function as a positive-feedback system and contribute to a worse prognosis. Therefore, physicians should pay more attention to patients with liver dysfunction in clinical practice.

Voriconazole is metabolized by enzymes that predominantly include CYP2C19, CYP3A4, and CYP2C9, [6, 48] and theoretically, the concomitant use of inducers or inhibitors of these drug-metabolizing enzymes should impact the pharmacokinetics of voriconazole. Unsurprisingly, concomitant medications were tested as a potential covariate in most of the included population pharmacokinetic studies, and a series of drugs were identified in the final model. Dolton et al. demonstrated that concomitant use of rifampicin (203\%), phenytoin (203\%), and St John's wort (107\%) significantly increased the value of $V_{\max }$, whereas short-term concomitant use of ritonavir decreased the value of $V_{\max }$ (42.9\%) [12]. Similarly, Pascual et al. [20] reported that the coadministration of rifampicin significantly increased the voriconazole CL by three-fold in adult patients with invasive mycoses. The impact of these agents on the pharmacokinetics of voriconazole was sufficiently large that the therapeutic range was not reached in most patients. Therefore, concomitant use of these agents is contraindicated as instructed in the prescribing information. In fact, several population pharmacokinetic studies [15, 16, 19, 25] did not enroll patients who received agents that substantially affect voriconazole exposure.
Compared with the above-mentioned agents, the coadministration of voriconazole with proton pump inhibitors (PPIs) and glucocorticoids was more common in clinical practice. Theoretically, glucocorticoids, which are considered CYP450 inducers, can decrease voriconazole exposure, and PPIs, which are CYP450 inhibitors, can increase voriconazole exposure. However, neither PPIs nor glucocorticoids appeared to influence the pharmacokinetics of voriconazole in the population pharmacokinetic analyses. For PPIs, only the study conducted by Mangal et al. [18] found that the Michaelis-Menten constant values increased by $79 \%$ when the drug was administered concomitantly with pantoprazole. The other population studies included in this review tested the concomitant use of PPIs as a covariate, but this covariate was not retained in the final model. Similarly, the concomitant use of glucocorticoids was tested as a potential covariate in numerous population pharmacokinetic studies, but only the study conducted by Dolton et al. [12] which involved 240 patients and 3352 observations, included glucocorticoids as a significant covariate in the model.

Overall, the impact of PPIs and glucocorticoids on the pharmacokinetics of voriconazole remains controversial. The absence of any significant effects of concomitantly used medications on the population parameters of voriconazole might be owing to the limited sample sizes and confounding factors. In addition, it should be mentioned that most of the included studies did not provide information regarding the type of specific agent and the dose applied. In fact, the results of many studies showed that voriconazole exposure was substantially influenced by both the type of PPI (or glucocorticoid) and the dose used [49-51]. Taking PPIs as an example, Cojutti et al. demonstrated that the impact of PPIs on voriconazole exposure exhibited varying magnitudes, as demonstrated by the following results (shown in descending order): pantoprazole $(80 \mathrm{mg})$, omeprazole $(80 \mathrm{mg})$, omeprazole $(40 \mathrm{mg})$, pantoprazole $(40 \mathrm{mg})$, and pantoprazole (20 mg) [51]. Thus, concomitantly used medications (particularly the various types and dosages of PPIs and glucocorticoids) should be tested in future population analyses.

Age was tested as a potential covariate in numerous studies, but only the study conducted by Wang et al. [21] included age as a significant covariate in the model. The association between the $\mathrm{CL}$ of voriconazole and age agrees with the fact that voriconazole is metabolized by drugmetabolizing enzymes and with the existence of a negative relationship between age and enzyme functional activity. Although other demographic covariates, such as sex, height, race, and body mass index, were tested, none were found to have a significant effect on the pharmacokinetic parameters in both adult and pediatric populations. According to the manufacturer, renal function has no influence on the pharmacokinetics of voriconazole. Unsurprisingly, the population pharmacokinetic analyses of voriconazole did not identify 
serum creatinine or creatinine CL as a significant biological covariate of the pharmacokinetics of voriconazole.

Although the above-mentioned covariates were incorporated in the population models, the pharmacokinetic variability remained relatively large. Thus, other potential covariates should be tested in model building in future studies. In recent years, numerous studies have reported that inflammation, which can be reflected by the C-reactive protein levels, might influence the voriconazole trough concentration [52-58]. A retrospective study revealed that despite similar voriconazole doses, the trough concentrations of voriconazole in patients with severe inflammation are significantly higher than those in patients with zero to moderate inflammation. For every $1-\mathrm{mg} / \mathrm{L}$ increase in the $\mathrm{C}$-reactive protein value, the voriconazole trough concentration is elevated by $0.015 \mathrm{mg} / \mathrm{L}$ [52].

Moreover, a significant negative correlation between the C-reactive protein value and the metabolic rate of voriconazole was detected in a retrospective study [53]. These findings can be explained by the negative regulation of various drug-metabolizing enzymes by proinflammatory cytokines, particularly interleukin- 6 and tumor necrosis factor- $\alpha$. The inflammatory state might play a significant role in the high variability in the pharmacokinetics of voriconazole and should be tested as a potential covariate in future population pharmacokinetic models.

With regard to model evaluation, external evaluation is considered the most stringent method for model testing and is beneficial for subsequent implementation in the management of voriconazole dosing. Unfortunately, only one included study [14] performed an external evaluation using a separate cohort. Thus, external evaluations of previously published models and comparisons of the predictive performance of the published models should be performed. In the majority of the included studies, simulation analyses were also performed to determine the optimal dosing regimens, and the recommended dosing strategies significantly varied between the different studies (or populations). Therefore, extrapolation of the dosing strategies to a specific population should be performed with caution.

\section{Conclusion}

This systematic review summarizes the relevant information for both clinicians and researchers on the population pharmacokinetics of voriconazole. For clinicians, this review highlights relevant predictors that can be considered for optimization of the voriconazole dose. Body weight, the CYP2C19 genotype, liver function, and concomitant medications are the most important factors associated with the variability in the pharmacokinetics of voriconazole. Understanding these factors and identifying subpopulations with special features could help improve the individualized dosing of voriconazole. Given the high inter- and intraindividual variability in the pharmacokinetics of voriconazole, TDM remains a suitable method for identifying inappropriate exposure. Most of the studies included in this review retained the CYP2C19 genotype as a significant covariate in the final model. Therefore, genetic testing should be encouraged if appropriate in clinical practice.

For researchers, further population pharmacokinetic studies on pediatric patients aged younger than 2 years are warranted. Moreover, several potential or controversial covariates, such as inflammation, the CYP3A4 genotype, concomitant medications (particularly PPIs and glucocorticoids), and various measures of body weight (IBW and ABW), should be tested because the unexplained variability remains relatively high. In addition, the previously published models should be externally evaluated, and the predictive performances of the models should be compared.

\section{Compliance with Ethical Standards}

Funding This work was supported by the Zhejiang Provincial Program for the Cultivation of High-Level Innovative Health Talents (Grant No. 2010-190-4), the Clinical Pharmacy of Zhejiang Medical Key Discipline (Grant No. 2018-2-3), and the Clinical Pharmacy of Hangzhou Medical Key Discipline (Grant No. 2017-68-7).

Conflict of interest Changcheng Shi, Yubo Xiao, Yong Mao, Jing Wu, and Nengming Lin have no conflicts of interest that are directly relevant to the content of this review.

OpenAccess This article is distributed under the terms of the Creative Commons Attribution-NonCommercial 4.0 International License (http://creativecommons.org/licenses/by-nc/4.0/), which permits any noncommercial use, distribution, and reproduction in any medium, provided you give appropriate credit to the original author(s) and the source, provide a link to the Creative Commons license, and indicate if changes were made.

\section{References}

1. Johnson LB, Kauffman CA. Voriconazole: a new triazole antifungal agent. Clin Infect Dis. 2003;36(5):630-7.

2. Patterson TF, Thompson GR 3rd, Denning DW, Fishman JA, Hadley $\mathrm{S}$, Herbrecht $\mathrm{R}$, et al. Practice guidelines for the diagnosis and management of aspergillosis: 2016 update by the Infectious Diseases Society of America. Clin Infect Dis. 2016;63(4):e1-60.

3. Pappas PG, Kauffman CA, Andes DR, Clancy CJ, Marr KA, Ostrosky-Zeichner L, et al. Clinical practice guideline for the management of candidiasis: 2016 update by the Infectious Diseases Society of America. Clin Infect Dis. 2016;62(4):e1-50.

4. Dolton MJ, McLachlan AJ. Voriconazole pharmacokinetics and exposure-response relationships: assessing the links between exposure, efficacy and toxicity. Int J Antimicrob Agents. 2014;44(3):183-93.

5. Elewa H, El-Mekaty E, El-Bardissy A, Ensom MH, Wilby KJ. Therapeutic drug monitoring of voriconazole in the management 
of invasive fungal infections: a critical review. Clin Pharmacokinet. 2015;54(12):1223-35.

6. Theuretzbacher U, Ihle F, Derendorf H. Pharmacokinetic/pharmacodynamic profile of voriconazole. Clin Pharmacokinet. 2006;45(7):649-63.

7. Park WB, Kim NH, Kim KH, Lee SH, Nam WS, Yoon SH, et al. The effect of therapeutic drug monitoring on safety and efficacy of voriconazole in invasive fungal infections: a randomized controlled trial. Clin Infect Dis. 2012;55(8):1080-7.

8. Kiang TK, Sherwin CM, Spigarelli MG, Ensom MH. Fundamentals of population pharmacokinetic modelling: modelling and software. Clin Pharmacokinet. 2012;51(8):515-25.

9. Thomson AH, Whiting B. Bayesian parameter estimation and population pharmacokinetics. Clin Pharmacokinet. 1992;22(6):447-67.

10. Sherwin CM, Kiang TK, Spigarelli MG, Ensom MH. Fundamentals of population pharmacokinetic modelling: validation methods. Clin Pharmacokinet. 2012;51(9):573-90.

11. Chen W, Xie H, Liang F, Meng D, Rui J, Yin X, et al. Population pharmacokinetics in China: the dynamics of intravenous voriconazole in critically ill patients with pulmonary disease. Biol Pharm Bull. 2015;38(7):996-1004.

12. Dolton MJ, Mikus G, Weiss J, Ray JE, McLachlan AJ. Understanding variability with voriconazole using a population pharmacokinetic approach: implications for optimal dosing. J Antimicrob Chemother. 2014;69(6):1633-41.

13. Han K, Capitano B, Bies R, Potoski BA, Husain S, Gilbert $\mathrm{S}$, et al. Bioavailability and population pharmacokinetics of voriconazole in lung transplant recipients. Antimicrob Agents Chemother. 2010;54(10):4424-31.

14. Han K, Bies R, Johnson H, Capitano B, Venkataramanan R. Population pharmacokinetic evaluation with external validation and Bayesian estimator of voriconazole in liver transplant recipients. Clin Pharmacokinet. 2011;50(3):201-14.

15. Li ZW, Peng FH, Yan M, Liang W, Liu XL, Wu YQ, et al. Impact of CYP2C19 genotype and liver function on voriconazole pharmacokinetics in renal transplant recipients. Ther Drug Monit. 2017;39(4):422-8.

16. Lin XB, Li ZW, Yan M, Zhang BK, Liang W, Wang F, et al. Population pharmacokinetics of voriconazole and CYP2C19 polymorphisms for optimizing dosing regimens in renal transplant recipients. Br J Clin Pharmacol. 2018;84(7):1587-97.

17. Liu P, Mould DR. Population pharmacokinetic analysis of voriconazole and anidulafungin in adult patients with invasive aspergillosis. Antimicrob Agents Chemother. 2014;58(8):4718-26.

18. Mangal N, Hamadeh IS, Arwood MJ, Cavallari LH, Samant TS, Klinker KP, et al. Optimization of voriconazole therapy for the treatment of invasive fungal infections in adults. Clin Pharmacol Ther. 2018;104(5):957-65.

19. Nomura K, Fujimoto Y, Kanbayashi Y, Ikawa K, Taniwaki M. Pharmacokinetic-pharmacodynamic analysis of voriconazole in Japanese patients with hematological malignancies. Eur J Clin Microbiol Infect Dis. 2008;27(11):1141-3.

20. Pascual A, Csajka C, Buclin T, Bolay S, Bille J, Calandra T, et al. Challenging recommended oral and intravenous voriconazole doses for improved efficacy and safety: population pharmacokinetics-based analysis of adult patients with invasive fungal infections. Clin Infect Dis. 2012;55(3):381-90.

21. Wang T, Chen S, Sun J, Cai J, Cheng X, Dong H, et al. Identification of factors influencing the pharmacokinetics of voriconazole and the optimization of dosage regimens based on Monte Carlo simulation in patients with invasive fungal infections. J Antimicrob Chemother. 2014;69(2):463-70.

22. Gastine S, Lehrnbecher T, Müller C, Farowski F, Bader P, Ullmann-Moskovits $\mathrm{J}$, et al. Pharmacokinetic modeling of voriconazole to develop an alternative dosing regimen in children. Antimicrob Agents Chemother. 2018;62(1):e01194-17.

23. Karlsson MO, Lutsar I, Milligan PA. Population pharmacokinetic analysis of voriconazole plasma concentration data from pediatric studies. Antimicrob Agents Chemother. 2009;53(3):935-44.

24. Muto C, Shoji S, Tomono Y, Liu P. Population pharmacokinetic analysis of voriconazole from a pharmacokinetic study with immunocompromised Japanese pediatric subjects. Antimicrob Agents Chemother. 2015;59(6):3216-23.

25. Walsh TJ, Karlsson MO, Driscoll T, Arguedas AG, Adamson P, Saez-Llorens X, et al. Pharmacokinetics and safety of intravenous voriconazole in children after single- or multiple-dose administration. Antimicrob Agents Chemother. 2004;48(6):2166-72.

26. Friberg LE, Ravva P, Karlsson MO, Liu P. Integrated population pharmacokinetic analysis of voriconazole in children, adolescents, and adults. Antimicrob Agents Chemother. 2012;56(6):3032-42.

27. de Velde F, Mouton JW, de Winter BCM, van Gelder T, Koch BCP. Clinical applications of population pharmacokinetic models of antibiotics: challenges and perspectives. Pharmacol Res. 2018;134:280-8.

28. Hope WW. Population pharmacokinetics of voriconazole in adults. Antimicrob Agents Chemother. 2012;56(1):526-31.

29. Neely M, Margol A, Fu X, van Guilder M, Bayard D, Schumitzky A, et al. Achieving target voriconazole concentrations more accurately in children and adolescents. Antimicrob Agents Chemother. 2015;59(6):3090-7.

30. McDougall DA, Martin J, Playford EG, Green B. Determination of a suitable voriconazole pharmacokinetic model for personalised dosing. J Pharmacokinet Pharmacodyn. 2016;43(2):165-77.

31. Kadam RS, Van Den Anker JN. Pediatric clinical pharmacology of voriconazole: role of pharmacokinetic/pharmacodynamic modeling in pharmacotherapy. Clin Pharmacokinet. 2016;55(9):1031-43.

32. Liu L, Zhou X, Wu T, Jiang H, Yang S, Zhang Y. Dose optimisation of voriconazole with therapeutic drug monitoring in children: a single-centre experience in China. Int J Antimicrob Agents. 2017;49(4):483-7.

33. Farkas A, Daroczi G, Villasurda P, Dolton M, Nakagaki M, Roberts JA. Comparative evaluation of the predictive performances of three different structural population pharmacokinetic models to predict future voriconazole concentrations. Antimicrob Agents Chemother. 2016;60(11):6806-12.

34. Gilljam M, Chaparro C, Tullis E, Chan C, Keshavjee S, Hutcheon M. GI complications after lung transplantation in patients with cystic fibrosis. Chest. 2003;123(1):37-41.

35. Bunnapradist S, Neri L, Wong W, Lentine KL, Burroughs TE, Pinsky BW, et al. Incidence and risk factors for diarrhea following kidney transplantation and association with graft loss and mortality. Am J Kidney Dis. 2008;51(3):478-86.

36. Zane NR, Thakker DR. A physiologically based pharmacokinetic model for voriconazole disposition predicts intestinal first-pass metabolism in children. Clin Pharmacokinet. 2014;53(12):1171-82.

37. Yanni SB, Annaert PP, Augustijns P, Ibrahim JG, Benjamin DK $\mathrm{Jr}$, Thakker DR. In vitro hepatic metabolism explains higher clearance of voriconazole in children versus adults: role of CYP2C19 and flavin-containing monooxygenase 3. Drug Metab Dispos. 2010;38(1):25-31.

38. Zane NR, Chen Y, Wang MZ, Thakker DR. Cytochrome P450 and flavin-containing monooxygenase families: age-dependent differences in expression and functional activity. Pediatr Res. 2018;83(2):527-35.

39. Upreti VV, Wahlstrom JL. Meta-analysis of hepatic cytochrome P450 ontogeny to underwrite the prediction of pediatric 
pharmacokinetics using physiologically based pharmacokinetic modeling. J Clin Pharmacol. 2016;56(3):266-83.

40. Davies-Vorbrodt S, Ito JI, Tegtmeier BR, Dadwal SS, Kriengkauykiat J. Voriconazole serum concentrations in obese and overweight immunocompromised patients: a retrospective review. Pharmacotherapy. 2013;33(1):22-30.

41. Pai MP, Lodise TP. Steady-state plasma pharmacokinetics of oral voriconazole in obese adults. Antimicrob Agents Chemother. 2011;55(6):2601-5.

42. Koselke E, Kraft S, Smith J, Nagel J. Evaluation of the effect of obesity on voriconazole serum concentrations. J Antimicrob Chemother. 2012;67(12):2957-62.

43. Moriyama B, Obeng AO, Barbarino J, Penzak SR, Henning SA, Scott SA, et al. Clinical Pharmacogenetics Implementation Consortium (CPIC) guidelines for CYP2C19 and voriconazole Therapy. Clin Pharmacol Ther. 2016;102(1):45-51.

44. Gautier-Veyret E, Fonrose X, Stanke-Labesque F. Pharmacogenetics of voriconazole: CYP2C19 but also CYP3A4 need to be genotyped. Clin Pharmacol Ther. 2017;102(2):189.

45. Duflot T, Schrapp A, Bellien J, Lamoureux F. Impact of CYP3A4 genotype on voriconazole exposure. Clin Pharmacol Ther. 2018;103(2):185-6.

46. He HR, Sun JY, Ren XD, Wang TT, Zhai YJ, Chen SY, et al. Effects of CYP3A4 polymorphisms on the plasma concentration of voriconazole. Eur J Clin Microbiol Infect Dis. 2015;34(4):811-9.

47. Luong ML, Al-Dabbagh M, Groll AH, Racil Z, Nannya Y, Mitsani D, et al. Utility of voriconazole therapeutic drug monitoring: a meta-analysis. J Antimicrob Chemother. 2016;71(7):1786-99.

48. Hyland R, Jones BC, Smith DA. Identification of the cytochrome P450 enzymes involved in the N-oxidation of voriconazole. Drug Metab Dispos. 2003;31(5):540-7.

49. Dolton MJ, Ray JE, Chen SC, Ng K, Pont LG, McLachlan AJ. Multicenter study of voriconazole pharmacokinetics and therapeutic drug monitoring. Antimicrob Agents Chemother. 2012;56(9):4793-9.
50. Qi F, Zhu L, Li N, Ge T, Xu G, Liao S. Influence of different proton pump inhibitors on the pharmacokinetics of voriconazole. Int J Antimicrob Agents. 2017;49(4):403-9.

51. Cojutti P, Candoni A, Forghieri F, Isola M, Zannier ME, Bigliardi $\mathrm{S}$, et al. Variability of voriconazole trough levels in haematological patients: influence of comedications with cytochrome P450(CYP) inhibitors and/or with CYP inhibitors plus CYP inducers. Basic Clin Pharmacol Toxicol. 2016;118(6):474-9.

52. van Wanrooy MJ, Span LF, Rodgers MG, van den Heuvel ER, Uges DR, van der Werf TS, et al. Inflammation is associated with voriconazole trough concentrations. Antimicrob Agents Chemother. 2014;58(12):7098-101.

53. Encalada Ventura MA, Span LF, van den Heuvel ER, Groothuis GM, Alffenaar JW. Influence of inflammation on voriconazole metabolism. Antimicrob Agents Chemother. 2015;59(5):2942-3.

54. Veringa A, Ter Avest M, Span LF, van den Heuvel ER, Touw DJ, Zijlstra JG, et al. Voriconazole metabolism is influenced by severe inflammation: a prospective study. J Antimicrob Chemother. 2017;72(1):261-7.

55. Encalada Ventura MA, van Wanrooy MJ, Span LF, Rodgers MG, van den Heuvel ER, Uges DR, et al. Longitudinal analysis of the effect of inflammation on voriconazole trough concentrations. Antimicrob Agents Chemother. 2016;60(5):2727-31.

56. Yasu T, Konuma T, Kato S, Kurokawa Y, Takahashi S, Tojo A. Serum C-reactive protein levels affect the plasma voriconazole trough levels in allogeneic hematopoietic cell transplant recipients. Leuk Lymphoma. 2017;58(11):2731-3.

57. Gautier-Veyret E, Truffot A, Bailly S, Fonrose X, Thiebaut-Bertrand A, Tonini J, et al. Inflammation is a potential risk factor of voriconazole overdose in hematological patients. Fundam Clin Pharmacol. 2018. https://doi.org/10.1111/fcp.12422. [Epub ahead of print].

58. Vreugdenhil B, van der Velden WJFM, Feuth T, Kox M, Pickkers P, van de Veerdonk FL, et al. Moderate correlation between systemic IL-6 responses and CRP with trough concentrations of voriconazole. Br J Clin Pharmacol. 2018;84(9):1980-8. 